\title{
An approach based on the LOWHM and induced LOWHM operators to group decision making under linguistic information
}

\author{
Jin Han Park ${ }^{1}$, Bu Young Lee ${ }^{2}$ and Mi Jung Son ${ }^{2}$ \\ ${ }^{1}$ Division of Applied Mathematics, Pukyong National University, Pusan 608-737, Korea \\ 2 Department of Mathematics, Dong-A University, Pusan 604-714, Korea
}

\begin{abstract}
In this paper, we propose an induced linguistic ordered weighted harmonic mean (ILOWHM) operator. The ILOWHM operator is more general type of aggregation operator, which is based on the LHM and LOWHM operators. Based on the LOWHM and ILOWHM operator, we develop an approach to group decision making with linguistic preference relations. Finally, an application of the approach to group decision making problem with linguistic preference relations is pointed out.
\end{abstract}

Key words : Group decision making, linguistic variable, linguistic preference relation, ILOWHM operator.

\section{Introduction}

Decision making problems generally consist of finding the most desirable alternative(s) from a given alternative set. The increasing complexity of the socio-economic environment makes it less and less possible for single decision maker to consider all relevant aspects of a problem [13]. As a result, many decision making processes, in the real world, take place in group settings. Group decision making problems follow a common resolution scheme composed by the following two phases:

(1) Aggregation phase: It combines the individual preferences to obtain a collective preference.

(2) Exploitation phase: It orders the collective preference values to obtain the best alternative(s).

Recently, a number of studies have focused on the group decision making with linguistic preference relations $[2,4,5,6,7,8,9,10,11,12,18,19,20]$. Herrera et al. [8] developed a consensus model for group decision making under linguistic assessments. It is based on the use of linguistic preferences to provide individuals' opinions, and on the use of fuzzy majority of consensus, represented by means of linguistic quantifier. Herrera et al. $[9,10]$ combined the linguistic ordered weighted averaging (LOWA) operator with linguistic preference relations and the concept of dominance and nondominance to show its

This paper was supported by Dong-A University Research Fund in 2007. Corresponding Author: Bu Young Lee

접수일자 : 2010년 3월 4일

완료일자 : 2010년 3월 25일 use in the field of group decision making, and presented three models of group decision making based on LOWA operator, and presented a consensus model in complete linguistic framework for group decision making. Herrera and Herrera-Viedma [6] analyzed the steps to follow in linguistic decision analysis of group decision making problem with linguistic preference relations. In some situations, however, the decision makers either are willing to provide only uncertain linguistic information, or take the input arguments as the form of uncertain linguistic variables rather than numerical ones because of time pressure, lack of knowledge, or data, and their limited expertise related to the problem domain. So, based induced ordered weighted averaging (IOWA) operator proposed Yager and Filev [25], Xu [20] introduced induced uncertain linguistic ordered weighted averaging (IULOWA) operator which take as their argument pair, called ULOWA pair, in which one component is used to induce an ordering over the second components which are given in the form of uncertain linguistic variables, and applied the IULOWA operator to group decision making with uncertain linguistic information. Xu [19] proposed some aggregation operators including the uncertain linguistic geometric mean (ULGM) operator, uncertain linguistic weighted geometric mean (ULWGM) operator, and induced uncertain linguistic ordered weighted geometric (IULOWG) operator, and developed an approach to group decision making with uncertain multiplicative linguistic relation. Information aggregation is 
essential process of gathering relevant information from multiple sources. Many techniques, such as the max and min operators, the weighted arithmetic average (WAA) operator, the weighted geometric mean operator, the weighted harmonic mean (WHM) operator, the ordered weighted averaging (OWA) operator, and so on have been developed to aggregate data information [16, 17, 21, 22, 23, 24, 26]. Harmonic mean is a conservative average to be used to provide for aggregation lying between the max and min operators. Harmonic mean is widely used to aggregate central tendency data. Consider that, in the existing literature, the harmonic mean is generally considered as a fusion technique of numerical data, in the real-life situations, the input data sometimes cannot be obtained exactly, but linguistic data can be given. Therefore, "how to aggregate linguistic data by using the harmonic mean?" is an interesting research topic and is worth paying attention to. Recently, Park et al. [14] developed some linguistic harmonic mean (LHM) operators, such as linguistic weighted harmonic mean (LWHM) operator, linguistic ordered weighted harmonic mean (LOWHM) operator and linguistic hybrid harmonic mean (LHHM) operator, and presented an approach to group decision making based on the developed operators. In this paper, we propose a new aggregation operator called induced LOWHM (ILOWHM) operator, and develop an approach, based on the LOWHM and ILOWHM operators, to group decision making with linguistic preference relations. Finally, an illustrative example is pointed out.

\section{Linguistic variables and operational laws}

Let $S=\left\{s_{i}: i=1,2, \ldots, t\right\}$ be a finite and totally ordered discrete term set. Any label, $s_{i}$, represents a possible value for a linguistic variable, and it must have the following characteristics [8]:

(1) The set is ordered: $s_{i} \geq s_{j}$ if $i \geq j$;

(2) There is the negation operator: $\operatorname{neg}\left(s_{i}\right)=s_{j}$ such that $j=t+1-i$.

(3) Max operator: $\max \left(s_{i}, s_{j}\right)=s_{i}$ if $s_{i} \geq s_{j}$;

(4) Min operator: $\min \left(s_{i}, s_{j}\right)=s_{i}$ if $s_{i} \leq s_{j}$.

For example, $S$ can be defined so as its elements are uniformly distributed on a scale on which a total order is defined:

$$
\begin{aligned}
& S=\left\{s_{1}=\text { extremely poor, } s_{2}=\right.\text { very poor } \\
& s_{3}=\text { poor, } s_{4}=\text { slightly poor, } s_{5}=\text { fair }, \\
& s_{6}=\text { slightly good, } s_{7}=\text { good } \\
&\left.s_{8}=\text { very good, } s_{9}=\text { extremely good }\right\}
\end{aligned}
$$

To preserve all the given information, we extend the discrete term set $S$ to a continuous linguistic term set $\bar{S}=\left\{s_{\alpha}: s_{1} \leq s_{\alpha} \leq s_{t}, \alpha \in[1, t]\right\}$, where, if $s_{\alpha} \in S$, then we call $s_{\alpha}$ an original linguistic term, otherwise, we call $s_{\alpha}$ the virtual linguistic term [19]. The decision maker, in general, uses the original linguistic terms to evaluate alternatives, and the virtual linguistic terms can only appear in operations.

Consider any two linguistic variables $s_{\alpha}$ and $s_{\beta}$, then we define the operations $s_{\alpha} \oplus s_{\beta}, \lambda s_{\alpha}$ and $\frac{1}{s_{\alpha}}$ as follows:

(1) $s_{\alpha} \oplus s_{\beta}=\min \left\{s_{\alpha+\beta}, s_{t}\right\}$;

(2) $\lambda s_{\alpha}=s_{\lambda \alpha}$, where $\lambda \in[0,1]$;

(3) $\frac{1}{s_{\alpha}}=s_{\frac{1}{\alpha}}$.

\section{The LOWHM and ILOWHM operators}

Definition 3.1. (Yager [23]) An OWA operator of dimension $n$ is a mapping OWA : $R^{n} \rightarrow R$ that has an associated vector $w=\left(w_{1}, w_{2}, \ldots, w_{n}\right)^{T}$ such that $w_{j} \geq 0$ and $\sum_{j=1}^{n} w_{j}=1$. Furthermore,

$$
\operatorname{OWA}_{w}\left(a_{1}, a_{2}, \ldots, a_{n}\right)=\sum_{j=1}^{n} w_{j} b_{j}
$$

where $b_{j}$ is the $j$ th largest of $a_{i}(i=1,2, \ldots, n)$. Especially, if $w_{i}=1, w_{j}=0, j \neq i$, then $b_{n} \leq \operatorname{OWA}_{w}\left(a_{1}, a_{2}\right.$, $\left.\ldots, a_{n}\right)=b_{i} \leq b_{1}$; if $w=\left(\frac{1}{n}, \frac{1}{n}, \ldots, \frac{1}{n}\right)^{T}$, then

$$
\begin{aligned}
\operatorname{OWA}_{w}\left(a_{1}, a_{2}, \ldots, a_{n}\right) & =\frac{1}{n} \sum_{j=1}^{n} b_{j}=\frac{1}{n} \sum_{j=1}^{n} a_{j} \\
& =\operatorname{AA}\left(a_{1}, a_{2}, \ldots, a_{n}\right) .
\end{aligned}
$$

Definition 3.2. (Bullen et al. [1]) An OWHM operator of dimension $n$ is a mapping OWHM : $\left(R^{+}\right)^{n} \rightarrow R^{+}$that has an associated vector $w=\left(w_{1}, w_{2}, \ldots, w_{n}\right)^{T}$ such that $w_{j} \geq 0$ and $\sum_{j=1}^{n} w_{j}=1$. Furthermore,

$$
\mathrm{OWHM}_{w}\left(a_{1}, a_{2}, \ldots, a_{n}\right)=\frac{1}{\sum_{j=1}^{n} \frac{w_{j}}{b_{j}}},
$$

where $b_{j}$ is the $j$ th largest of the $a_{i}(i=1,2, \ldots, n)$. Especially, if $w_{i}=1, w_{j}=0, j \neq i$, then $b_{n} \leq \mathrm{OWHM}_{w}\left(a_{1}, a_{2}, \ldots, a_{n}\right)=b_{i} \leq b_{1}$; if $w=$ $\left(\frac{1}{n}, \frac{1}{n}, \ldots, \frac{1}{n}\right)^{T}$, then the OWHM operator is reduced to the harmonic mean (HM) operator, i.e.,

$$
\operatorname{HM}_{w}\left(a_{1}, a_{2}, \ldots, a_{n}\right)=\frac{n}{\sum_{j=1}^{n} \frac{1}{a_{j}}} .
$$


Definition 3.3. (Park et al. [14]) Let LWHM : $\bar{S}^{n} \rightarrow \bar{S}$, if

$$
\begin{aligned}
\operatorname{LWHM}_{w}\left(s_{\alpha_{1}}, s_{\alpha_{2}}, \ldots, s_{\alpha_{n}}\right) & \frac{1}{}=\frac{w_{1}}{s_{\alpha_{1}}} \oplus \frac{w_{2}}{s_{\alpha_{2}}} \oplus \cdots \oplus \frac{w_{n}}{s_{\alpha_{n}}} \\
& =\frac{1}{s_{\frac{w_{1}}{\alpha_{1}}} \oplus s \frac{w_{2}}{\alpha_{2}} \oplus \cdots \oplus s \frac{w_{n}}{\alpha_{n}}} \\
& =\frac{1}{s_{\sum_{j=1}^{n} \frac{w_{j}}{\alpha_{j}}}},
\end{aligned}
$$

where $w=\left(w_{1}, w_{2}, \ldots, w_{n}\right)^{T}$ is the weight vector of the $s_{\alpha_{j}}$ with $w_{j} \in[0,1], \sum_{j=1}^{n} w_{j}=1, s_{\alpha_{j}} \in \bar{S}$, then LWHM is called the linguistic weighted harmonic mean (LWHM) operator. Especially, if $w_{i}=1$ and $w_{j}=0$, $j \neq i$, then $\operatorname{LWHM}\left(s_{\alpha_{1}}, s_{\alpha_{2}}, \ldots, s_{\alpha_{n}}\right)=s_{\alpha_{i}}$; if $w=$ $\left(\frac{1}{n}, \frac{1}{n}, \ldots, \frac{1}{n}\right)^{T}$, then LWHM operator is called the linguistic harmonic mean (LHM) operator, i.e.,

$$
\operatorname{LHM}\left(s_{\alpha_{1}}, s_{\alpha_{2}}, \ldots, s_{\alpha_{n}}\right)=\frac{n}{s_{\sum_{j=1}^{n} \frac{1}{\alpha_{j}}}} .
$$

The OWHM operator can only be used in situations where the input arguments are the exact numerical variable. Recently, Park et al. [14] extended the OWGM operator to accommodate the situations where the input arguments are linguistic variables.

Definition 3.4. A LOWHM operator of dimension $n$ is a mapping LOWHM $: \bar{S}^{n} \rightarrow \bar{S}$, which has an associated vector $w=\left(w_{1}, w_{2}, \ldots, w_{n}\right)^{T}$ with $w_{j} \in[0,1]$ and $\sum_{j=1}^{n} w_{j}=1$, such that

$$
\begin{aligned}
\operatorname{LOWHM}_{w}\left(s_{\alpha_{1}}, s_{\alpha_{2}}, \ldots, s_{\alpha_{n}}\right) & 1 \\
& =\frac{w_{1}}{\frac{w_{1}}{s_{1}} \oplus \frac{w_{2}}{s_{\beta_{2}}} \oplus \cdots \oplus \frac{w_{n}}{s_{\beta_{n}}}} \\
& =\frac{1}{s \frac{w_{1}}{\beta_{1}} \oplus s \frac{w_{2}}{\beta_{2}} \oplus \cdots \oplus s \frac{w_{n}}{\beta_{n}}} \\
& =\frac{1}{s_{\sum_{j=1}^{n} \frac{w_{j}}{\beta_{j}}}},
\end{aligned}
$$

where $s_{\beta_{j}}$ is the $j$ th largest of the $s_{\alpha_{i}}$.

Especially, if there is a tie between $s_{\alpha_{i}}$ and $s_{\alpha_{j}}$, then we replace each of $s_{\alpha_{i}}$ and $s_{\alpha_{j}}$ by their average $\left(s_{\alpha_{i}} \oplus s_{\alpha_{j}}\right) / 2$ in the process of aggregation. If $k$ items are tied, then we replace these by $k$ replicas of their average. The weighted vector $w=\left(w_{1}, w_{2}, \ldots, w_{n}\right)^{T}$ can be determined by using some weight determining methods like the normal distribution based method.

Yager and Filev [25] introduced an induced ordered weighted averaging (IOWA) operator, which takes as its argument pairs, called OWA pairs, in which one component is used to induce an ordering over the second components, which are exact numerical values and then aggregated.
Definition 3.5. (Yager and Filov [25]) An IOWA operator is defined as follows:

$\operatorname{IOWA}_{w}\left(\left\langle u_{1}, a_{1}\right\rangle,\left\langle u_{2}, a_{2}\right\rangle, \ldots,\left\langle u_{n}, a_{n}\right\rangle\right)=\sum_{j=1}^{n} w_{j} b_{j}$,

where $w=\left(w_{1}, w_{2}, \ldots, w_{n}\right)^{T}$ is a weighting vector, such that $w_{j} \in[0,1], j=1,2, \ldots, n, \sum_{j=1}^{n} w_{j}=1, b_{j}$ is the $a_{i}$ value of the OWA pair $\left\langle u_{i}, a_{i}\right\rangle$ having the $j$ th largest $u_{i}$, and $u_{i}$ in $\left\langle u_{i}, a_{i}\right\rangle$ is referred to as the order inducing variable and $a_{i}$ as the argument variable, $a_{i} \in R^{+}$, $i=1,2, \ldots, n, R^{+}$is the set of all positive real numbers. Especially, if $w=\left(\frac{1}{n}, \frac{1}{n}, \ldots, \frac{1}{n}\right)^{T}$, then it is reduced to the AA operator; if $u_{i}=a_{i}$, for all $i$, then IOWA is reduced to the OWA operator; if $u_{i}=$ No. $i$, for all $i$, where No. $i$ is the ordered position of the $a_{i}$, then IOWA is the weighted arithmetic averaging operator.

In the following, we shall develop an induced LOWHM (ILOWHM) operator to accommodate the situations where the input arguments are linguistic variables.

Definition 3.6. An ILOWHM operator is defined as follows:

$$
\begin{aligned}
\operatorname{ILOWHM}_{w}\left(\left\langle u_{1}, s_{\alpha_{1}}\right\rangle,\left\langle u_{2}, s_{\alpha_{2}}\right\rangle, \ldots,\left\langle u_{n}, s_{\alpha_{n}}\right\rangle\right) & \frac{1}{\frac{w_{1}}{s_{\beta_{1}}} \oplus \frac{w_{2}}{s_{\beta_{2}}} \oplus \cdots \oplus \frac{w_{n}}{s_{\beta_{n}}}} \\
= & \frac{1}{s \frac{w_{1}}{\beta_{1}} \oplus s \frac{w_{2}}{\beta_{2}} \oplus \cdots \oplus s \frac{w_{n}}{\beta_{n}}} \\
& =\frac{1}{s_{\sum_{j=1}^{n} \frac{w_{j}}{\beta_{j}}}},
\end{aligned}
$$

where $w=\left(w_{1}, w_{2}, \ldots, w_{n}\right)^{T}$ is a weighting vector, such that $w_{j} \in[0,1], j=1,2, \ldots, n, \sum_{j=1}^{n} w_{j}=1, s_{\beta_{j}}$ is the $s_{\alpha_{i}}$ value of the LOWHM pair $\left\langle u_{i}, s_{\alpha_{i}}\right\rangle$ having the $j$ th largest $u_{i}$, and $u_{i}$ in $\left\langle u_{i}, s_{\alpha_{i}}\right\rangle$ is referred to as the order inducing variable and $s_{\alpha_{i}}$ as the linguistic argument variable. Especially, if $w=\left(\frac{1}{n}, \frac{1}{n}, \ldots, \frac{1}{n}\right)^{T}$, then ILOWHM is reduced to the LHM operator; if $u_{i}=s_{\alpha_{i}}$, for all $i$, then ILOWHM is reduced to the LOWHM operator; if $u_{i}=$ No. $i$, for all $i$, where No. $i$ is the ordered position of the $a_{i}$, then ILOWHM is the LWHM operator.

However, if there is a tie between $\left\langle u_{i}, s_{\alpha_{i}}\right\rangle,\left\langle u_{j}, s_{\alpha_{j}}\right\rangle$ with respect to order-inducing variables, in this case, we can follow the policy presented by Yager and Filov [25] to replace the arguments of the tied objects by the mean of the arguments of the tied objects (i.e., we replace the argument component of each of $\left\langle u_{i}, s_{\alpha_{i}}\right\rangle$ and $\left\langle u_{j}, s_{\alpha_{j}}\right\rangle$ by their average $\left(s_{\alpha_{i}} \oplus s_{\alpha_{j}}\right) / 2$ ). If $k$ items are tied, we replace these by $k$ replicas of their average.

In the following, we shall give two examples to specify the special cases with respect to the inducing variables. 
Example 3.7. Consider the following collection of LOWHM pairs:

$$
\left\langle s_{4}, s_{3}\right\rangle,\left\langle s_{6}, s_{7}\right\rangle,\left\langle s_{3}, s_{1}\right\rangle,\left\langle s_{5}, s_{4}\right\rangle .
$$

Performing the ordering the LOWHM pairs with respect to the first component, we have:

$$
\left\langle s_{6}, s_{7}\right\rangle,\left\langle s_{5}, s_{4}\right\rangle,\left\langle s_{4}, s_{3}\right\rangle,\left\langle s_{3}, s_{1}\right\rangle .
$$

This ordering induces the ordered linguistic arguments:

$$
s_{\beta_{1}}=s_{7}, s_{\beta_{2}}=s_{4}, s_{\beta_{3}}=s_{3}, s_{\beta_{4}}=s_{1} .
$$

If the weighting vector $w=(0.3,0.1,0.4,0.2)^{T}$, then we get an aggregated value:

$$
\begin{aligned}
\operatorname{ILOWHM}_{w}\left(\left\langle s_{4}, s_{3}\right\rangle,\left\langle s_{5}, s_{7}\right\rangle,\left\langle s_{3}, s_{1}\right\rangle,\left\langle s_{6}, s_{4}\right\rangle\right) \\
=\frac{1}{\frac{0.3}{s_{7}} \oplus \frac{0.1}{s_{4}} \oplus \frac{0.4}{s_{3}} \oplus \frac{0.2}{s_{1}}} \\
=\frac{1}{s_{\frac{0.3}{7}} \oplus s_{\frac{0.1}{4}} \oplus s_{\frac{0.4}{3}} \oplus s_{\frac{0.2}{1}}} \\
=s_{2.49} .
\end{aligned}
$$

Example 3.8. Consider the following collection of LOWHM pairs:

$$
\left\langle\text { No. } 3, s_{3}\right\rangle,\left\langle\text { No. } 2, s_{7}\right\rangle,\left\langle\text { No. } 4, s_{1}\right\rangle,\left\langle\text { No. } 1, s_{4}\right\rangle \text {. }
$$

Performing the ordering the LOWHM pairs with respect to the first component, we have:

$$
\left\langle\text { No. } 1, s_{4}\right\rangle,\left\langle\text { No. } 2, s_{7}\right\rangle,\left\langle\text { No. } 3, s_{3}\right\rangle,\left\langle\text { No. } 4, s_{1}\right\rangle \text {. }
$$

This ordering induces the ordered linguistic arguments:

$$
s_{\beta_{1}}=s_{4}, s_{\beta_{2}}=s_{7}, s_{\beta_{3}}=s_{3}, s_{\beta_{4}}=s_{1} .
$$

If the weighting vector $w=(0.3,0.1,0.4,0.2)^{T}$, then we get an aggregated value:

$$
\begin{aligned}
\operatorname{ILOWHM}_{w}\left(\left\langle\mathrm{No}_{0}, s_{3}\right\rangle\right. & \left.,\left\langle\mathrm{No.}_{2}, s_{7}\right\rangle,\left\langle\mathrm{No.}_{1}, s_{1}\right\rangle,\left\langle\text { No.1, } s_{4}\right\rangle\right) \\
& =\frac{1}{\frac{0.3}{s_{4}} \oplus \frac{0.1}{s_{7}} \oplus \frac{0.4}{s_{3}} \oplus \frac{0.2}{s_{1}}} \\
& =\frac{1}{s_{\frac{0.3}{4}} \oplus s_{\frac{0.1}{7}} \oplus s_{\frac{0.4}{3}} \oplus s_{\frac{0.2}{1}}} \\
& =s_{2.36}
\end{aligned}
$$

The ILOWHM operator has many desirable properties similar to those of the IOWA operator [25]:

Theorem 3.9. (Commutativity)

$$
\begin{aligned}
\operatorname{ILOWHM}_{w}\left(s_{\alpha_{1}}, s_{\alpha_{2}}, \ldots, s_{\alpha_{n}}\right) & \\
& =\operatorname{ILOWHM}_{w}\left(s_{\alpha_{1}}^{\prime}, s_{\alpha_{2}}^{\prime}, \ldots, s_{\alpha_{n}}^{\prime}\right),
\end{aligned}
$$

where $\left(s_{\alpha_{1}}^{\prime}, s_{\alpha_{2}}^{\prime}, \ldots, s_{\alpha_{n}}^{\prime}\right)$ is a permutation of $\left(s_{\alpha_{1}}, s_{\alpha_{2}}\right.$, $\left.\ldots, s_{\alpha_{n}}\right)$.
Theorem 3.10. (Idempotency) If $s_{\alpha_{j}}=s_{\alpha}$, for all $j$, then

$$
\operatorname{ILOWHM}_{w}\left(s_{\alpha_{1}}, s_{\alpha_{2}}, \ldots, s_{\alpha_{n}}\right)=s_{\alpha} .
$$

Theorem 3.11. (Monotonicity) If $s_{\alpha_{j}} \leq s_{\alpha_{j}}^{*}$, for all $j$, then

$$
\begin{aligned}
\operatorname{ILOWHM}_{w}\left(s_{\alpha_{1}}, s_{\alpha_{2}},\right. & \left.\ldots, s_{\alpha_{n}}\right) \\
& \leq \operatorname{ILOWHM}_{w}\left(s_{\alpha_{1}}^{*}, s_{\alpha_{2}}^{*}, \ldots, s_{\alpha_{n}}^{*}\right) .
\end{aligned}
$$

\section{An approach to group decision making with linguistic preference relations}

Consider a group decision making problem with linguistic preference information. Let $X=\left\{x_{1}, x_{2}, \ldots, x_{n}\right\}$ be the set of alternatives, and $D=\left\{d_{1}, d_{2}, \ldots, d_{m}\right\}$ be the set of decision makers. Let $v=\left(v_{1}, v_{2}, \ldots, v_{n}\right)^{T}$ be the weight vector of decision makers, where $v_{l} \geq 0$, $l=1,2, \ldots, m, \sum_{l=1}^{m} v_{l}=1$. The decision maker $d_{l} \in D$ compares these alternatives with respect to a single criterion by the linguistic terms in the set $\bar{S}=\left\{s_{\alpha}: s_{1} \leq\right.$ $\left.s_{\alpha} \leq s_{t}, \alpha \in[1, t]\right\}$, and constructs the linguistic preference relation $R_{l}=\left(r_{i j}^{(l)}\right)_{n \times n}$, whose element $r_{i j}^{(l)} \in \bar{S}$ estimates the preference degree of alternative $x_{i}$ over $x_{j}$, for all $l=1,2, \ldots, m ; i, j=1,2, \ldots, n$.

Theorem 4.1. Let $R_{1}, R_{2}, \ldots, R_{m}$ be the linguistic preferences provided by $m$ decision makers $d_{l}(i=1,2, \ldots, m)$, where $R_{l}=\left(r_{i j}^{(l)}\right)_{n \times n}, r_{i j}^{(l)} \in \bar{S}(l=1,2, \ldots, m ; i, j=$ $1,2, \ldots, n)$, then their collective linguistic preference relation $R=\left(r_{i j}\right)_{n \times n}$ is also linguistic preference relation with:

$$
\begin{aligned}
r_{i j} & =\operatorname{ILOWHM}_{w}\left(\left\langle v_{1}, r_{i j}^{(1)}\right\rangle,\left\langle v_{2}, r_{i j}^{(2)}\right\rangle, \ldots,\left\langle v_{m}, r_{i j}^{(m)}\right\rangle\right) \\
& =\frac{1}{\frac{w_{1}}{t_{i j}^{(1)}} \oplus \frac{w_{2}}{t_{i j}^{(2)}} \oplus \cdots \oplus \frac{w_{n}}{t_{i j}^{(m)}}},
\end{aligned}
$$

where $t_{i j}^{(k)}$ is the $r_{i j}^{(l)}$ value of the LOWHM pair $\left\langle v_{l}, r_{i j}^{(l)}\right\rangle$ having the $k$ th largest $v_{l}$, for all $l=1,2, \ldots, m ; i, j=$ $1,2, \ldots, n$.

In the following, we shall develop an approach based on the LOWHM and ILOWHM operators to group decision making with linguistic preference relations.

Step 1. For a group decision making problem with linguistic preference information. Let $X=\left\{x_{1}, x_{2}, \ldots, x_{n}\right\}$ be the set of alternatives, and $D=\left\{d_{1}, d_{2}, \ldots, d_{m}\right\}$ be the set of decision makers. Let $v=\left(v_{1}, v_{2}, \ldots, v_{n}\right)^{T}$ be the weight vector of decision makers, where $v_{l} \geq 0$, $l=1,2, \ldots, m, \sum_{l=1}^{m} v_{l}=1$. The decision maker $d_{l} \in D$ compares these alternatives with respect to a single criterion by the linguistic terms in the set $\bar{S}=\left\{s_{\alpha}: s_{1} \leq\right.$ $\left.s_{\alpha} \leq s_{t}, \alpha \in[1, t]\right\}$, and constructs the linguistic preference relation $R_{l}=\left(r_{i j}^{(l)}\right)_{n \times n}$, whose element $r_{i j}^{(l)} \in \bar{S}$ 
estimates the preference degree of alternative $x_{i}$ over $x_{j}$, for all $l=1,2, \ldots, m ; i, j=1,2, \ldots, n$.

Step 2. Utilize the ILOWHM operator:

$$
\begin{gathered}
r_{i j}=\operatorname{ILOWHM}_{w}\left(\left\langle v_{1}, r_{i j}^{(1)}\right\rangle,\left\langle v_{2}, r_{i j}^{(2)}\right\rangle, \ldots,\left\langle v_{m}, r_{i j}^{(m)}\right\rangle\right) \\
i, j=1,2, \ldots, n
\end{gathered}
$$

to aggregate all the linguistic preference relations $R_{l}=$ $\left(r_{i j}^{(l)}\right)_{n \times n}(l=1,2, \ldots, m)$ into a collective linguistic preference $R=\left(r_{i j}\right)_{n \times n}$, where $w=\left(w_{1}, w_{2}, \ldots, w_{n}\right)^{T}$ is weighting vector associated with the ILOWHM operator, such that $w_{j} \in[0,1], j=1,2, \ldots, n$, and $\sum_{j=1}^{n} w_{j}=1$.

Step 3. Utilize the LOWHM operator:

$$
r_{i}=\mathrm{LOWHM}_{w^{\prime}}\left(r_{i 1}, r_{i 2}, \ldots, r_{i n}\right), \quad i=1,2, \ldots, n
$$

to aggregate $r_{i j}$ corresponding to the alternative $x_{i}$, and then get the collective linguistic preference degree $r_{i}(i=$ $1,2, \ldots, n)$ of the $i$ th alternative over all the other alternatives, where $w^{\prime}=\left(w_{1}^{\prime}, w_{2}^{\prime}, \ldots, w_{n}^{\prime}\right)^{T}$ is the weighting vector associated the LOWHM operator, such that $w_{j}^{\prime} \in[0,1]$, $j=1,2, \ldots, n, \sum_{j=1}^{n} w_{j}^{\prime}=1$.

Step 4. Rank all the alternatives $x_{i}(i=1,2, \ldots, n)$ and select the best one(s) in accordance with the value of $r_{i}(i=1,2, \ldots, n)$.

Step 5. End.

\section{Illustrative example}

Let us suppose that there is an investment company, which wants to invest a sum of money in the best option (adapted from [7]). There is a panel with five possible alternatives in which to invest the money:

1) $x_{1}$ is a car industry.

2) $x_{2}$ is a food company.

3) $x_{3}$ is a computer company.

4) $x_{4}$ is an arms company.

5) $x_{5}$ is a TV company.

One main criterion used is growth analysis. There are three decision makers $d_{l}(l=1,2,3)$, whose weight vector $v=(0.2,0.5,0.3)^{T}$. The decision-makers compare these five schools with respect to the criterion growth analysis by using the linguistic terms in the set

$$
\begin{aligned}
& S=\left\{s_{1}=\text { extremely poor, } s_{2}=\right.\text { very poor } \\
& s_{3}=\text { poor, } s_{4}=\text { slightly poor, } s_{5}=\text { fair }, \\
& s_{6}=\text { slightly good, } s_{7}=\text { good, } \\
&\left.s_{8}=\text { very good, } s_{9}=\text { extremely good }\right\}
\end{aligned}
$$

and construct, respectively, the linguistic preference relations $R_{l}(l=1,2,3)$ as listed in Tables 1-3.
Table 1. The linguistic preference relation $R_{1}$

\begin{tabular}{cccccc}
\hline & $x_{1}$ & $x_{2}$ & $x_{3}$ & $x_{4}$ & $x_{5}$ \\
\hline$x_{1}$ & $s_{5}$ & $s_{2}$ & $s_{4}$ & $s_{3}$ & $s_{7}$ \\
$x_{2}$ & $s_{8}$ & $s_{5}$ & $s_{5}$ & $s_{4}$ & $s_{6}$ \\
$x_{3}$ & $s_{5}$ & $s_{5}$ & $s_{5}$ & $s_{2}$ & $s_{4}$ \\
$x_{4}$ & $s_{7}$ & $s_{6}$ & $s_{8}$ & $s_{5}$ & $s_{3}$ \\
$x_{5}$ & $s_{3}$ & $s_{4}$ & $s_{6}$ & $s_{7}$ & $s_{5}$
\end{tabular}

Table 2. The linguistic preference relation $R_{2}$

\begin{tabular}{cccccc}
\hline & $x_{1}$ & $x_{2}$ & $x_{3}$ & $x_{4}$ & $x_{5}$ \\
\hline$x_{1}$ & $s_{5}$ & $s_{3}$ & $s_{5}$ & $s_{6}$ & $s_{5}$ \\
$x_{2}$ & $s_{7}$ & $s_{5}$ & $s_{7}$ & $s_{2}$ & $s_{5}$ \\
$x_{3}$ & $s_{6}$ & $s_{3}$ & $s_{5}$ & $s_{4}$ & $s_{6}$ \\
$x_{4}$ & $s_{4}$ & $s_{5}$ & $s_{6}$ & $s_{5}$ & $s_{4}$ \\
$x_{5}$ & $s_{5}$ & $s_{6}$ & $s_{4}$ & $s_{6}$ & $s_{5}$ \\
\hline
\end{tabular}

Table 3. The linguistic preference relation $R_{3}$

\begin{tabular}{cccccc}
\hline & $x_{1}$ & $x_{2}$ & $x_{3}$ & $x_{4}$ & $x_{5}$ \\
\hline$x_{1}$ & $s_{5}$ & $s_{4}$ & $s_{6}$ & $s_{4}$ & $s_{8}$ \\
$x_{2}$ & $s_{3}$ & $s_{5}$ & $s_{4}$ & $s_{3}$ & $s_{4}$ \\
$x_{3}$ & $s_{4}$ & $s_{6}$ & $s_{5}$ & $s_{5}$ & $s_{7}$ \\
$x_{4}$ & $s_{6}$ & $s_{7}$ & $s_{5}$ & $s_{5}$ & $s_{2}$ \\
$x_{5}$ & $s_{8}$ & $s_{3}$ & $s_{3}$ & $s_{5}$ & $s_{5}$ \\
\hline
\end{tabular}

To get the most desirable alternative(s), the following steps are involved:

Step 1. Utilize the ILOWHM operator (let its weighting vector be $\left.w=(0.2,0.6,0.2)^{T}\right)$ :

$$
\begin{aligned}
r_{i j}= & \operatorname{ILOWHM}_{w}\left(\left\langle v_{1}, r_{i j}^{(1)}\right\rangle,\left\langle v_{2}, r_{i j}^{(2)}\right\rangle,\left\langle v_{3}, r_{i j}^{(3)}\right\rangle\right), \\
& i, j=1,2,3,4,5
\end{aligned}
$$

to aggregate all the linguistic preference relations $R_{l}=$ $\left(r_{i j}^{(l)}\right)_{5 \times 5}(l=1,2,3)$ into the collective linguistic preference relation $R=\left(r_{i j}\right)_{5 \times 5}$ as listed in Table 4 .

Table 4. The collective linguistic preference relation $R$

\begin{tabular}{cccccc}
\hline & $x_{1}$ & $x_{2}$ & $x_{3}$ & $x_{4}$ & $x_{5}$ \\
\hline$x_{1}$ & $s_{5.00}$ & $s_{3.16}$ & $s_{5.26}$ & $s_{4.00}$ & $s_{6.97}$ \\
$x_{2}$ & $s_{3.94}$ & $s_{5.00}$ & $s_{4.58}$ & $s_{2.86}$ & $s_{4.48}$ \\
$x_{3}$ & $s_{4.48}$ & $s_{4.84}$ & $s_{5.00}$ & $s_{3.70}$ & $s_{5.92}$ \\
$x_{4}$ & $s_{5.60}$ & $s_{6.29}$ & $s_{5.61}$ & $s_{5.00}$ & $s_{2.40}$ \\
$x_{5}$ & $s_{5.50}$ & $s_{3.53}$ & $s_{3.53}$ & $s_{5.50}$ & $s_{5.00}$ \\
\hline
\end{tabular}


Step 2. Utilize the LOWHM operator (let its weighting vector be $\left.w^{\prime}=(0.15,0.2,0.3,0.2,0.15)^{T}\right)$ :

$r_{i}=\mathrm{LOWHM}_{w^{\prime}}\left(r_{i 1}, r_{i 2}, r_{i 3}, r_{i 4}, r_{i 5}\right), \quad i=1,2, \ldots, n$

to aggregate $r_{i j}(j=1,2,3,4,5)$ corresponding to the alternative $x_{i}$, and then get the collective linguistic preference degree $r_{i}$ of the $i$ th alternative over all the other alternatives:

$$
\begin{aligned}
& r_{1}=s_{4.61}, r_{2}=s_{4.10}, r_{3}=s_{4.71}, r_{4}=s_{4.64}, \\
& r_{5}=s_{4.49} .
\end{aligned}
$$

Step 3: Utilize the values of $r_{i}(i=1,2,3,4,5)$ to rank the alternatives:

$$
x_{3} \succ x_{4} \succ x_{1} \succ x_{5} \succ x_{2}
$$

and thus the most desirable alternative is $x_{3}$.

\section{Conclusions}

In this paper, we have developed an approach to group decision making with linguistic preference relations. First, we have utilized the ILOWHM operator to aggregate the individual linguistic preference relations into a collective linguistic preference relation, and then utilized the LOWHM operator to aggregate the collective preference to get the collective linguistic preference degrees. Finally, the collective linguistic preference degrees have been used to rank all the given alternatives. Theoretical analysis and numerical results have shown that the developed approach is straightforward and has no loss of information.

\section{References}

[1] P.S. Bullen, D.S. Mitrinovi and P.M. Vasi, Means and their inequalities, The Netherlands, Reidel, 1988.

[2] M. Delgado, J.L. Verdegay and M.A. Vila, "Linguistic decision making models," Int. J. Intell. Syst., vol. 8, pp. 351-370, 1993.

[3] J.C. Harsanyi, "Cardinal welfare, individualistic ethics, and interpersonal comparisons of utility," $J$. Polit. Economy., vol. 63, pp. 309-321, 1955.

[4] F. Herrera and E. Herrera-Viedma, "Aggregation operators for linguistic weighted information," IEEE Trans. Syst. Man Cybern., vol. 27, pp. 646-656, 1997.

[5] F. Herrera and E. Herrera-Viedma, "Choice functions and mechanisms for linguistic preference relations," Eur. J. Oper. Res., vol. 120, pp. 144-161, 2000.
[6] F. Herrera and E. Herrera-Viedma, "Linguistic decision analysis: steps for solving decision problems under linguistic information," Fuzzy Sets Syst., vol. 115, pp. 67-82, 2000.

[7] F. Herrera, E. Herrera-Viedma and L. Martinez, "A fusion approach for managing multi-granularity linguistic term sets in decision making," Fuzzy Sets Syst., vol. 114, pp. 43-58, 2000.

[8] F. Herrera, E. Herrera-Viedma and J.L. Verdegay, “A model of consensus in group decision making under linguistic assessments," Fuzzy Sets Syst., vol. 78, pp. 73-87, 1996.

[9] F. Herrera, E. Herrera-Viedma and J.L. Verdegay, "Direct approach process in group decision making using linguistic OWA operators," Fuzzy Sets Syst., vol. 79, pp. 175-190, 1996.

[10] F. Herrera, E. Herrera-Viedma and J.L. Verdegay, “A rational consensus model in group decision making linguistic assessments," Fuzzy Sets Syst., vol. 88, pp. 31-49, 1997.

[11] F. Herrera and L. Martinez, "A 2-tuple fuzzy linguistic representation model for computing with words," IEEE Trans. Fuzzy Syst., vol. 8, pp. 746-752, 2000.

[12] F. Herrera and J.L. Verdegay, "Linguistic assessments in group decision," Proceedings of 11th European Congress of Fuzzy Intelligent Technology, pp 941948, 1993.

[13] S.H. Kim, S.H. Choi and J.K. Kim, "An intractive procedure for multiple attribute group decision making with incomplete information: range-based approach," Eur. J. Oper. Res., vol. 118, pp. 139-152, 1999.

[14] J.H. Park, M.G. Gwak and Y.C. Kwun, "Linguistic harmonic mean operators and their applications to group decision making," submitted.

[15] J.H. Park, Y.C. Kwun and J.S. Park, "Multiple attribute group decision making problems based on fuzzy number intuitionistic fuzzy information," J. Korean Institute Intell. Sys., vol. 19, pp. 265-272, 2009.

[16] V. Torra and Y. Narukawa, Information fusion and aggregating information, Berlin, Springer, 2007.

[17] Z.S. Xu, Uncertain Multiple Attribute Decision Making: Methods and Applications, Tsinghua University Press, Beijing, 2004.

[18] Z.S. Xu, "A method based on linguistic aggregation operators for group decision making with linguistic preference relations," Inf. Sci., vol. 166, pp. 19-30, 2004. 
[19] Z.S. Xu, "An approach based on the uncertain LOWG and induced uncertain LOWG operators to group decision making with uncertain multiplicative linguistic preference relations," Decis. Support Syst., vol. 41, pp. 488-499, 2006.

[20] Z.S. Xu, "Induced uncertain linguistic OWA operators applied to group decision making," Inf. Fusion, vol. 7, pp. 231-238, 2006.

[21] Z.S. Xu and Q.L. Da, "The uncertain OWA operators," Int. J. Intell. Syst., vol. 17, pp. 569-575, 2002.

[22] Z.S. Xu and Q.L. Da, "An overview of operators for aggregationg information," Int. J. Intell. Syst., vol. 18, pp. 953-969, 2003.

[23] R.R. Yager, "On ordered weighted averaging aggregation operators in multicriteria decision making," IEEE Trans. Syst. Man Cybern., vol. 18, pp. 183-190, 1988.

[24] R.R. Yager, "Families and extension of OWA aggregation,” Fuzzy Sets Syst., vol. 59, pp. 125-148, 1993.

[25] R.R. Yager and D.O. Filev, "Induced ordered weighted averaging operators," IEEE Trans. Syst. Man Cybern., vol. 29, pp. 141-150, 1999.
[26] R.R. Yager and J. Kacprzyk, The ordered weighted averaging operator: Theory and application, Boston, Kluwer, 1997.

\section{저 자 소 개}

\section{Jin Han Park}

Professor of Pukyong National University

Research Area: Fuzzy Mathematics, Fuzzy Topology,

General Topology, Decision Making

E-mail : jihpark@pknu.ac.kr

\section{Bu Young Lee}

Professor of Dong-A University

Research Area: Fuzzy Mathematics, Fuzzy topology, Decision Making

E-mail : bylee@dau.ac.kr

\section{Mi Jung Son}

Professor of Korea Maritime University

Research Area: Fuzzy Mathematics, Fuzzy Topology

E-mail : mjson72@korea.com 